\title{
Efficacy of 3D conformal thoracic radiotherapy for extensive-stage small-cell lung cancer: A retrospective study
}

\author{
ZUPENG LUAN $^{1-3^{*}}$, ZHIWU WANG ${ }^{1,2^{*}}$, WEI HUANG ${ }^{2}$, JIAN ZHANG $^{2}$, \\ WEI DONG ${ }^{1,2}$, WEI ZHANG ${ }^{1,2}$, BAOSHENG $\mathrm{LI}^{2}$, TAO ZHOU ${ }^{2}$, HONGSHENG LI ${ }^{2}$, \\ ZICHENG ZHANG ${ }^{2}$, ZHONGTANG WANG ${ }^{2}$, HONGFU SUN $^{3}$ and YAN YI $^{2}$ \\ ${ }^{1}$ Department of Radiation Oncology, Cancer Hospital, Tianjin Medical University, \\ National Clinical Research Center for Cancer, Key Laboratory if Cancer Prevention and Therapy, Tianjin 300060; \\ ${ }^{2}$ Department of Radiation Oncology, Shandong Cancer Hospital, Shandong Academy of Medical Sciences, Jinan, \\ Shandong 250117; ${ }^{3}$ Department of Radiation Oncology, Jinan Third People's Hospital, Jinan, Shandong 250101, P.R. China
}

Received July 25, 2014; Accepted April 2, 2015

DOI: $10.3892 / \mathrm{etm} .2015 .2526$

\begin{abstract}
The aim of the present study was to evaluate the effect of 3-dimensional conformal thoracic radiotherapy (TRT) on extensive-stage small-cell lung cancer (ES-SCLC). A total of 165 patients with ES-SCLC were enrolled in the present study, including 82 patients receiving chemotherapy combined with TRT (the ChT/TRT group) and 83 patients receiving chemotherapy alone (the ChT group). The overall survival (OS) and progression-free survival (PFS) rates were compared between the ChT/TRT and ChT groups, and the prognostic factors for OS rate were identified. It was found that the patients had a median OS time of 15 months, and 2- and 5-year OS rates of 31.5 and $2.4 \%$, respectively. The $2-$ and 5 -year OS rates were 35.3 and $2.4 \%$ in the ChT/TRT group, and 14.5 and $2.4 \%$ in the ChT group, respectively $(\mathrm{P}<0.05)$. The 1 - and 2 -year PFS rates were 35.4 and $6.0 \%$ in the ChT/TRT group, and 20.5 and $6.0 \%$ in the ChT group, respectively $(\mathrm{P}<0.05)$. The median PFS was 11 months in the 20 patients receiving TRT at $45 \mathrm{~Gy} / 30$ fractions twice daily, and 9 months in the 22 patients receiving TRT at $60 \mathrm{~Gy} / 30$ fractions daily $(\mathrm{P}=0.043)$. Multivariate analysis revealed that receiving $\geq 4$ cycles of chemotherapy $(\mathrm{P}=0.001)$ and TRT $(\mathrm{P}=0.008)$ were favorable prognostic factors for OS. It was concluded that the addition of TRT improves the OS and PFS rates of patients with ES-SCLC, and TRT administration at $45 \mathrm{~Gy} / 30$ fractions twice daily is feasible and tolerable for the
\end{abstract}

Correspondence to: Dr Baosheng Li, Department of Radiation Oncology, Shandong Cancer Hospital, Shandong Academy of Medical Sciences, 440 Jiyan Road, Jinan, Shandong 250117, P.R. China

E-mail: baoshli@yahoo.com

*Contributed equally

Key words: extensive-stage small-cell lung cancer, 3-dimensional conformal thoracic radiotherapy, chemotherapy, efficacy treatment of ES-SCLC. Thus, TRT and receiving $\geq 4$ cycles of chemotherapy are independent, favorable prognostic factors for OS in patients with ES-SCLC.

\section{Introduction}

Lung cancer is a leading cause of cancer-associated mortality worldwide, with $>1$ million mortalities annually (1). Small-cell lung cancer (SCLC) accounts for $10-15 \%$ of lung cancer cases (2). It is estimated that $70 \%$ of patients with SCLC have extensive-stage SCLC (ES-SCLC) at the time of diagnosis (3).

Currently, the first-line therapy for ES-SCLC remains dependent on platinum-based chemotherapy with cisplatin-etoposide (EP) (4). The addition of thoracic radiotherapy (TRT) is suggested in certain patients due to its confirmed value in the improvement of the overall survival (OS) rate $(5,6)$. Although SCLC is sensitive to chemotherapy and radiotherapy, with a $60-80 \%$ response rate, the median survival rate of patients with ES-SCLC is estimated to be only 7-12 months following 4-6 cycles of standard chemotherapy (4).

Systemic chemotherapy in combination with concurrent TRT have been demonstrated to be effective in the treatment of limited-stage SCLC (LS-SCLC) by improving the progression-free survival (PFS) and OS rates (7-9). The National Comprehensive Cancer Network (NCCN) guidelines recommend that TRT should be initiated during the first or second cycles of chemotherapy in cases of LS-SCLC at 45 Gy administered twice per day in 1.5 Gy fractions, or 60-70 Gy in 2 Gy daily fractions (10-12). However, the effect of TRT on ES-SCLC remains unknown. The present retrospective study evaluated the effect of 3-dimensional (3D) conformal TRT on the clinical outcome of patients with ES-SCLC.

\section{Materials and methods}

Subjects. A total of 165 patients with histologically or cytologically confirmed ES-SCLC, enrolled at the Shandong Cancer Hospital (Jinan, China) from January 2005 to 
December 2008, were involved in the present study. The patients included 140 males and 25 females, with a median age of 60 years (range, 26-87 years). The subjects were assigned to two groups. Subjects in the ChT group $(n=83)$ underwent chemotherapy only, while those in the ChT/TRT group $(n=82)$ were given chemotherapy concurrently with TRT.

Therapy. The chemotherapy schedule consisted of the administration of etoposide $100 \mathrm{mg} / \mathrm{m}^{2}$ on days $1-5$, combined with cisplatin $30 \mathrm{mg} / \mathrm{m}^{2}$ on days $1-3$ or carboplatin $300 \mathrm{mg} / \mathrm{m}^{2}$ on day 1.3D-conformal TRT was performed following 1-6 cycles of chemotherapy at a dose of $1.5 \mathrm{~Gy} /$ fraction twice per day or $2 \mathrm{~Gy} /$ fraction daily, with a total dose of 40-62 Gy.

The target volume was defined according to the preoperative radiotherapy response assessment. If a stable disease (SD) was achieved, the gross target volume (GTV) included the primary tumor and the positive lymph node. If a complete response (CR) was achieved, the GTV included the primary tumor bed and the locations of the positive lymph node, which were diagnosed by computed tomography (CT) scanning prior to chemotherapy. If a progressive disease (PD) occurred, the GTV included the primary tumor, the positive lymph node and the new lesions. The clinical target volume (CTV) was defined as the GTV plus a $7 \mathrm{~mm}$ margin, while the planning target volume (PTV) was defined as the CTV plus a $10 \mathrm{~mm}$ margin. Prophylactic cranial irradiation (PCI) was administered to the 5 patients who achieved $\mathrm{CR}$.

Assessment of response to therapy. All patients underwent a physical examination, electrocardiography, blood chemistry analyses, brain magnetic resonance imaging (MRI), thoracic and abdominal CT scans, and radionuclide bone imaging prior to treatment. All examinations, with the exception of radionuclide bone imaging, were repeated every 2 cycles of chemotherapy or prior to TRT, and then every 3 months until 2 years, followed by every 3-6 months.

The response to the therapy was assessed using the Response Evaluation Criteria in Solid Tumors (13). A CR was defined as the complete disappearance of all objective evidence of disease for $\geq 4$ weeks; a partial response (PR) was defined as $\geq 50 \%$ reduction in the sum of the longest diameter (LD) of target lesions lasting $\geq 4$ weeks, taking as reference the baseline sum LD and without the appearance of any new lesions. PD was defined as the development of new lesions or $a \geq 20 \%$ increase in the sum of the LD of the target lesions; SD was defined as neither sufficient reduction to qualify for PR nor sufficient increase to qualify for PD lasting $\geq 6$ weeks, taking as reference the smallest sum LD (13).

Evaluation of therapy-associated toxicity. Toxic effects associated with the therapy, including leukopenia, thrombocytopenia, anemia, nausea and vomiting, were assessed according to the National Cancer Institute Common Toxicity Criteria (NCI-CTC) version 3.0. Radiation-induced pneumonitis and esophagitis were evaluated in the ChT/TRT group only.

Ethical consideration. The present study was approved by the Ethics Review Committee of Shandong Cancer Hospital.
Informed consent was obtained from the patients or their relatives prior to their participation, with a detailed description of the potential benefits of the study.

Statistical analysis. Differences in proportions were tested for statistical significance using the $\chi^{2}$ test. The Kaplan-Meier method was used to estimate OS and PFS. Factors used in multivariate survival analysis included gender, age, Karnofsky Performance Status (KPS) score, smoking status, TRT and the number of chemotherapy cycles. A Cox proportional hazards model was employed to identify significant variables. The associations between clinical factors and OS rate were evaluated using univariate survival analysis with the Kaplan-Meier method. All statistical analyses were performed using SPSS software, version 18.0 (SPSS, Inc., Chicago, IL, USA). P<0.05 was considered to indicate a statistically statistical significant difference.

\section{Results}

Patient characteristics. All patients underwent a follow-up subsequent to therapy, with a median period of 43.2 months (range, 27-73 months). In the ChT/TRT group, 64 cases had metastasis in one organ, 17 cases had metastasis in two organs, and 1 case had metastasis in three organs; metastasis to bone was detected in 31 patients, brain metastasis in 17 patients, lung metastasis in 16 patients, liver metastasis in 13 patients, adrenal gland metastasis in 17 patients and metastasis to other organs in 7 patients. In the ChT group, 47 patients had metastasis in one organ, 33 patients had metastasis in two organs, and 3 patients had metastasis in three organs; bone metastasis was detected in 33 cases, brain metastasis in 21 cases, lung metastasis in 18 cases, liver metastasis in 18 cases, adrenal gland metastasis in 19 cases and there was metastasis to other organs in 13 cases. There were no significant differences in gender, age, KPS score, smoking status and the number of chemotherapy cycles between the ChT and ChT/TRT groups $(\mathrm{P}>0.05)$. However, a significant difference was detected in the number of metastatic organs between the two groups $(\mathrm{P}=0.005$; Table I).

Response to therapy. Table II lists the therapies administered to the 165 patients, including the 82 individuals undergoing chemotherapy and TRT, and the 83 individuals undergoing chemotherapy alone. A total of 124 cases $(75.2 \%)$ responded to the therapy. There were 69 cases $(84.1 \%)$ responsive to therapy in the ChT/TRT group, including 12 cases achieving a CR. There were 55 cases $(66.2 \%)$ responsive in the ChT group, including 9 cases achieving a CR. The initial chemotherapy prior to TRT resulted in CR in 2 cases, PR in 68 patients, SD in 11 cases and PD in 1 patient in the ChT/TRT group. The timing of TRT with relation to the course of chemotherapy is also presented in Table II.

Whole-brain radiotherapy was administered to 38 patients in the ChT/TRT group and 26 patients in the ChT group. The 64 cases with bone metastasis received bisphosphonate treatment at the beginning of therapy. PCI was administered to the 5 patients with CR. Additional chemotherapy or palliative radiotherapy was given to 67 patients in the ChT/TRT group and 62 patients in the ChT group with PD, while 6 patients in 
Table I. Comparison of the demographic and clinical characteristics of patients with extensive-stage small-cell lung cancer in the ChT/TRT and ChT groups.

\begin{tabular}{|c|c|c|c|c|}
\hline Characteristic & Total & ChT/TRT group (\%) & ChT group (\%) & P-value \\
\hline Gender, $\mathrm{n}$ & & & & 0.836 \\
\hline Male & 138 & $68(82.9)$ & $70(84.3)$ & \\
\hline Female & 27 & $14(17.1)$ & $13(15.7)$ & \\
\hline Age & & & & 0.339 \\
\hline Range, years & $26-87$ & $26-83$ & $36-87$ & \\
\hline Median, years & 59 & 55 & 64 & \\
\hline$<60$ years, $n$ & 79 & $54(65.9)$ & $25(30.1)$ & \\
\hline$\geq 60$ years, $n$ & 86 & $28(34.1)$ & $58(69.9)$ & \\
\hline KPS score, $n$ & & & & 0.704 \\
\hline$\geq 80$ & 131 & $64(78)$ & $67(80.7)$ & \\
\hline$<80$ & 34 & $18(22)$ & $16(19.3)$ & \\
\hline Smoking status, $\mathrm{n}$ & & & & 0.522 \\
\hline Yes & 102 & $53(64.6)$ & $49(59)$ & \\
\hline No & 63 & $29(35.4)$ & $34(41)$ & \\
\hline No. of ChT cycles & & & & 0.355 \\
\hline$<4$ & 20 & $8(9.8)$ & $12(14.5)$ & \\
\hline$\geq 4$ & 145 & $74(90.2)$ & $71(85.5)$ & \\
\hline No. of metastatic organs & & & & 0.005 \\
\hline 1 & 111 & 64 & 47 & \\
\hline$\geq 2$ & 54 & 18 & 36 & \\
\hline
\end{tabular}

ChT/TRT, chemotherapy combined with thoracic radiotherapy; ChT, chemotherapy; KPS, Karnofsky performance status.

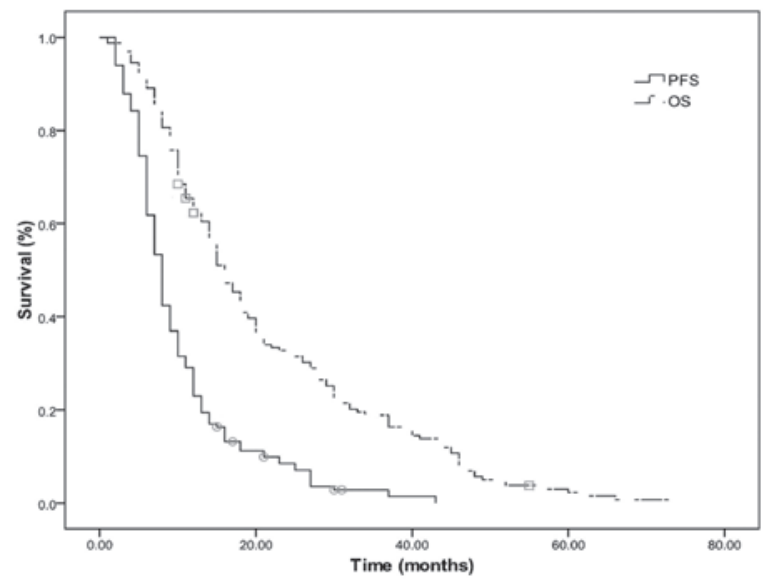

Figure 1. Overall survival (OS) and progression-free survival (PFS) rates of all subjects with extensive-stage small-cell lung cancer.

the ChT/TRT group and 9 patients in the ChT group accepted supportive care, which included nutritional support and symptomatic treatment.

Survival rate. The median OS and PFS times were 15 and 8 months, respectively, and the median 2- and 5-year OS rates were 31.5 and $2.4 \%$, respectively (Fig. 1). The median OS and PFS times were 18 and 9 months in the ChT/TRT group, with median 2- and 5-year OS rates of 35.3 and $2.4 \%$, and 1- and 2-year PFS rates of 35.4 and $6.0 \%$, respectively. In the ChT group, the median OS and PFS times were 12 and 6 months, with median 2- and 5-year OS rates of 14.5 and 2.4\% $(\mathrm{P}=0.033$; Fig. 2A) and 1- and 2-year PFS rates of 20.5 and $6.0 \%$, respectively $(\mathrm{P}=0.011$; Fig. $2 \mathrm{~B})$.

The median OS and PFS times were 17 and 8 months in the 129 patients receiving $\geq 4$ cycles of chemotherapy, and 10 and 6 months in the 36 patients receiving $<4$ cycles of chemotherapy (all $\mathrm{P}=0.001$; Fig. 3). The median PFS was 11 months in the 20 patients undergoing 45 Gy radiotherapy at $1.5 \mathrm{~Gy} /$ fraction twice per day, and 9 months in the 22 patients undergoing 60 Gy 
Table II. Initial treatment of patients with extensive-stage small-cell lung cancer in the ChT/TRT and ChT groups.

\begin{tabular}{llc}
\hline & ChT/TRT & ChT \\
Treatment & group (n) & group (n) \\
\hline
\end{tabular}

No. of ChT cycles

prior to TRT

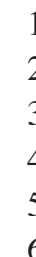

$$
\begin{aligned}
& 1 \\
& 2 \\
& 3 \\
& 4 \\
& 5
\end{aligned}
$$$$
2
$$$$
3
$$$$
6
$$

No. of initial ChT cycles

3
4
5
6
7
8

ChT regimen

EP

CE

Response to ChT

prior to TRT

CR
PR
SD
PD

TRT dose (Gy)

40

42

44

45

46

48

50

52

54

56

58

60

62

Radiation dosing

$1.5 \mathrm{~Gy} /$ fraction BID 22

2 Gy/fraction QD

PCI 30 Gy

WBRT

1

\section{5}

1

4

22

1

4

12

3

3

5

1

20

1

Bone RT

ChT, chemotherapy; TRT, thoracic radiation therapy; Gy, grays; EP, combined cisplatin and etoposide; CE, combined carboplatin and etoposide; CR, complete response; PR, partial response; SD, stable disease; $\mathrm{PD}$, progressive disease; BID, twice daily; QD, daily; PCI, prophylactic cranial irradiation; RT, radiotherapy; WBRT, whole-brain radiotherapy. radiotherapy at $2 \mathrm{~Gy} /$ fraction daily $(\mathrm{P}=0.043)$. No significant differences were observed in the OS and PFS rates between the patients receiving $\geq 6$ and $<6$ cycles of chemotherapy ( $P>0.05$ ). Furthermore, there were no significant differences in the OS and PFS rates among the patients receiving 2, 4 or 6 cycles of chemotherapy prior to TRT in the ChT/TRT group (all P>0.05). In patients with metastasis in 1 organ, the median OS time, 2- and 5-year OS rates were 18 months, 32.8 and 3.1\% in the ChT/TRT group, respectively, whereas they were 15 months, 48.9 and $2.1 \%$ in the ChT group, respectively (Fig. 4).

Prognostic factors. Univariate survival analysis revealed that KPS score, age at diagnosis, gender, smoking status, and number of metastatic organs had no association with OS rate; however, TRT and $\geq 4$ cycles of chemotherapy were found to correlate with survival (Table III). Multivariate analysis revealed that receiving $\geq 4$ cycles of chemotherapy $(\mathrm{P}=0.001)$ and TRT $(\mathrm{P}=0.008)$ were favorable prognostic factors for OS (Table IV).

Treatment failure. SCLC progression and distant metastasis were observed in 23 and 39 patients, respectively, in the ChT/TRT group, and 41 and 35 patients, respectively, in the ChT group. A higher local control of SCLC was observed in the ChT/TRT compared with the ChT group (48.8 vs. 28\%; $\mathrm{P}=0.006$ ), while no significant difference in the distant control was found between the two groups $(\mathrm{P}>0.05)$.

Therapy-associated toxicity. Therapy-induced toxicity was evaluated in the 165 eligible patients. Leukopenia occurred more frequently in the ChT/TRT group than in the ChT group, while no significant differences were detected in the occurrence of other toxicities. Subgroup analyses revealed that there was a higher occurrence of leukopenia in the patients receiving 45 Gy radiotherapy at $1.5 \mathrm{~Gy} /$ fraction twice per day compared with those receiving $60 \mathrm{~Gy}$ radiotherapy at $2 \mathrm{~Gy} /$ fraction daily. Grade 2 esophagitis was observed in 2 patients and pneumonitis was reported in 3 patients (grade 2 pneumonitis in 2 patients; grade 3 pneumonitis in 1 patient) in the ChT/TRT group (Table V).

\section{Discussion}

Although much progress has been achieved in the treatment of lung cancer, it remains the leading cause of cancer-associated mortality throughout the world, and is characterized by an extremely high mortality rate (14). There are two types of lung cancer: SCLC accounts for 10-15\% of all lung cancers, while non-small-cell lung cancer (NSCLC) accounts for $\sim 85 \%$ of all lung cancers (15). Untreated SCLC follows an aggressive course, with a median survival time of 2-4 months (16). Despite its generally late presentation and high risk of dissemination, SCLC is extremely sensitive to chemotherapy and radiotherapy (17). Chemotherapy is the mainstay of treatment for SCLC, while radiation therapy that involves the use of high energy X-rays focused on the specific disease site to directly destroy cancer cells, is usually recommended during chemotherapy to relieve pain and prevent and cure brain metastases (17-21).

Combined chemotherapy has been the standard treatment for ES-SCLC for almost 30 years and the administration of 
A

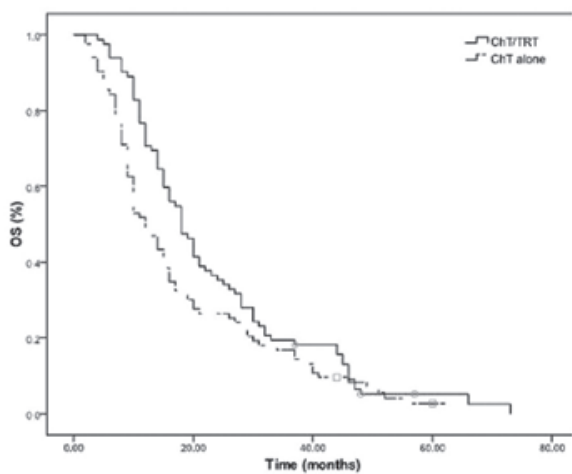

B

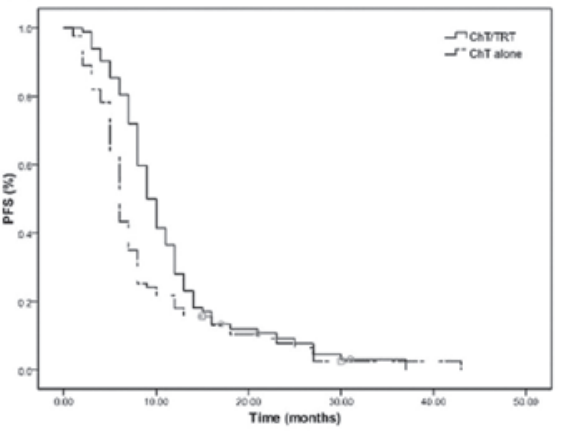

Figure 2. (A) Overall survival (OS) and (B) progression-free survival (PFS) rates of the patients with extensive-stage small-cell lung cancer in the chemotherapy combined with thoracic radiation therapy (ChT/TRT) and chemotherapy (ChT) groups.

A

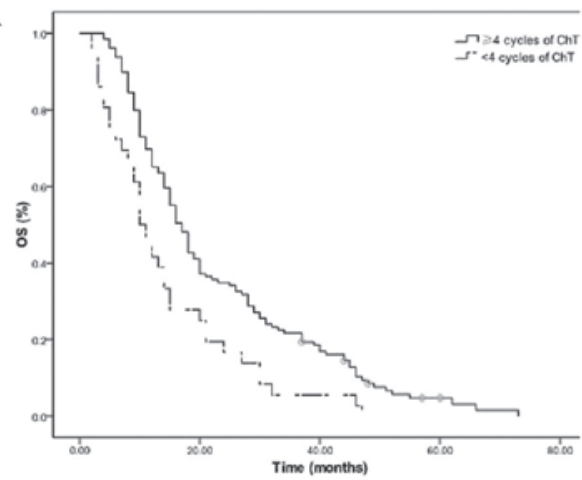

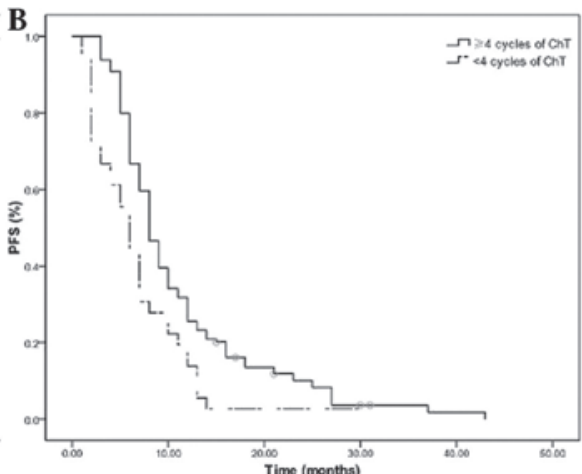

Figure 3. (A) Overall survival (OS) and (B) progression-free survival (PFS) rates of the patients with extensive-stage small-cell lung cancer receiving $\geq 4$ cycles and $<4$ cycles of chemotherapy $(\mathrm{ChT})$.

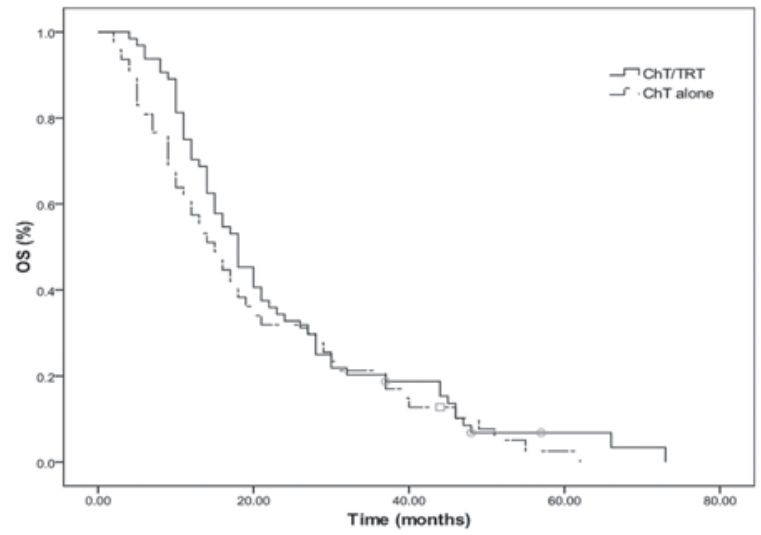

Figure 4. Overall survival (OS) rate of patients with extensive-stage small-cell lung cancer with metastasis in 1 organ in the chemotherapy combined with thoracic radiation therapy (ChT/TRT) and chemotherapy (ChT) groups.

carboplatin or cisplatin with etoposide remains the standard of care for patients with ES-SCLC (4). In the American College of Chest Physicians (ACCP), NCCN and the European Society for Medical Oncology (ESMO) guidelines, patients with ES-SCLC are advised to receive 4-6 cycles of cisplatin- or carboplatin-based combination chemotherapy (such as cisplatin plus etoposide or irinotecan) $(16,22,23)$. Although EP remains the most widely used combination, a randomized trial that compared the combination of cisplatin with either etoposide or irinotecan in ES-SCLC demonstrated that the irinotecan-cispl- atin combination (IP) was superior to EP. The median survival time was 12.8 months in patients treated with IP vs. 9.4 months in patients treated with the EP combination; the 2-year survival rate was also superior at $19.5 \%$ for IP vs. 5.2\% for EP (24). However, a confirmatory study in the United States failed to show the superiority of either regimen (25). Although the EP and IP combinations have shown comparable OS rates and survival outcomes, the IP combination has been found to cause increased gastrointestinal toxicity (26). Generally, radiotherapy is only used in patients with ES-SCLC to palliate symptoms if required 
Table III. Univariate analysis of the prognostic factors for survival in patients with extensive-stage small-cell lung cancer.

\begin{tabular}{|c|c|c|c|c|c|}
\hline Characteristic & $\begin{array}{l}\text { Mean survival } \\
\text { (months) }\end{array}$ & 2-year OS, \% & 5-year OS, \% & $\chi^{2}$ & P-value \\
\hline Gender & & & & 0.052 & 0.820 \\
\hline Male & 15 & 31.3 & 2.2 & & \\
\hline Female & 14 & 30.8 & 3.8 & & \\
\hline Age, years & & & & 2.590 & 0.108 \\
\hline$<60$ & 18 & 34.2 & 3.8 & & \\
\hline$\geq 60$ & 14 & 26.7 & 1 & & \\
\hline KPS score & & & & 0.056 & 0.813 \\
\hline$\geq 80$ & 16 & 32.3 & 1.6 & & \\
\hline$<80$ & 12 & 27.3 & & & \\
\hline Smoking status & & & & 2.127 & 0.145 \\
\hline Yes & 15 & 26.5 & 0 & & \\
\hline No & 15 & 34.9 & 4.8 & & \\
\hline No. of ChT cycles & & & & 10.527 & 0.001 \\
\hline$<4$ & 10 & 16.7 & 0 & & \\
\hline$\geq 4$ & 17 & 34.1 & 3.1 & & \\
\hline Treatment & & & & 4.534 & 0.033 \\
\hline ChT/TRT & 18 & 35.3 & 2.4 & & \\
\hline ChT alone & 12 & 14.5 & 2.4 & & \\
\hline No. of metastatic organs & & & & 2.435 & 0.119 \\
\hline 1 & 17 & 32.4 & 2.7 & & \\
\hline$\geq 2$ & 12 & 25.9 & 1.9 & & \\
\hline
\end{tabular}

OS, overall survival; KPS, Karnofsky performance status; ChT, chemotherapy; ChT/TRT, chemotherapy combined with thoracic radiation therapy.

(such as for painful bone metastases). Response rates are high; however, patients invariably relapse (27). The ACCP guidelines suggest that consolidative TRT to the chest is a treatment option for patients who achieve a CR outside the chest and CR or PR in the chest (16). However, to the best of our knowledge, the present study is the first study to examine the efficacy of TRT for the treatment of ES-SCLC.

In the current study, $75.2 \%$ of the total individuals studied responded to therapy; of these, $84.1 \%$ of the subjects in the ChT/TRT group responded and $66.2 \%$ of the subjects in the ChT group responded. The ES-SCLC patients receiving chemotherapy and 3D conformal TRT had a median OS time of 18 months, and 2- and 5-year OS rates of 35.3 and $2.4 \%$, respectively. The patients receiving chemotherapy alone had a median OS time of 12 months, and 2- and 5-year OS rates of 14.5 and $2.4 \%$, respectively (all $\mathrm{P}<0.05$ ). The median PFS times were 9 and 6 months in the ChT/TRT and ChT groups, respectively, while the 1- and 2-year PFS rates were 35.4 and $6.0 \%$ in the ChT/TRT group, and 20.5 and $6.0 \%$ in the ChT group, respectively. Furthermore, multivariate analysis showed that receiving $\geq 4$ cycles of chemotherapy and TRT were independent, favorable prognostic factors for OS.

A previous study investigated the efficacy and toxicity of EP chemotherapy with or without accelerated hyperfractionated radiotherapy and combined daily carboplatin/etoposide (CE) in patients with EC-SCLC. In patients with a CR or PR following 3 cycles of standard EP, it was found that patients who subsequently received thoracic radiotherapy with 54 Gy in 36 fractions over 18 treatment days in combination with CE followed by two cycles of EP had a significantly improved survival rate compared with that of patients given an additional 4 cycles of EP. Local control was improved in the radiotherapy group, although not significantly. No difference in distant metastasis-free survival was observed between the groups, and acute high-grade toxicity was higher in the patients that received chemotherapy. Thus, the addition of accelerated hyperfractionated radiotherapy to the treatment of the most favorable subset of patients led to improved survival over that obtained with chemotherapy alone (28). The present results also suggest that TRT may be a beneficial treatment for patients with ES-SCLC.

The selection of radiotherapy dose is a critical factor that determines its efficacy. Currently, TRT delivered at $60 \mathrm{~Gy} / 30$ fractions daily is more widely used for ES-SCLC treatment compared with $45 \mathrm{~Gy} / 30$ fractions twice per day due to convenience, a higher effective dose and the shorter duration of the treatment course (29). The two radiotherapy regimens have been recommended for the treatment of LS-SCLC; however, significant differences have been reported in the toxicity, disease control and survival rates between the two regimens (30). A phase III trial comparing daily with twice-daily radiotherapies for LS-SCLC showed 
Table IV. Multivariate analysis of the prognostic factors for survival in patients with extensive-stage small-cell lung cancer.

\begin{tabular}{lccc}
\hline Characteristic & HR & 95\% CI & P-value \\
\hline Gender (female vs. male) & 1.096 & $0.693-1.732$ & 0.696 \\
Age, years (<60 vs. $\geq 60)$ & 1.023 & $0.719-1.454$ & 0.901 \\
KPS score (<80 vs. $\geq 80)$ & 0.999 & $0.655-1.493$ & 0.958 \\
Smoking status (yes vs. no) & 0.836 & $0.608-1.203$ & 0.368 \\
No. of ChT cycles (<4 vs. $\geq 4)$ & 0.440 & $0.279-0.712$ & 0.001 \\
Treatment $($ ChT alone vs. ChT/TRT) & 0.607 & $0.418-0.880$ & 0.008 \\
No. of ChT cycles $(<6$ vs. $\geq 6)$ & 1.006 & $0.673-1.468$ & 0.975 \\
No. of metastasis organs $(>2$ vs. 1) & 0.791 & $0.563-1.108$ & 0.172 \\
\hline
\end{tabular}

HR, hazard ratio; CI, confidence interval; KPS, Karnofsky performance status; ChT, chemotherapy; ChT/TRT, chemotherapy combined with thoracic radiation therapy.

Table V. Incidence of toxic effects in the ChT/TRT and ChT groups.

\begin{tabular}{lccc}
\hline & \multicolumn{2}{c}{ Group (n) } & \\
\cline { 2 - 3 } Toxic effect/grade & ChT/TRT & ChT & P-value \\
\hline Hematologic toxicity grade $\geq 3$ & & & \\
Leukopenia & 47 & 29 & 0.004 \\
Thrombocytopenia & 6 & 9 & 0.431 \\
Anemia & 7 & 6 & 0.755 \\
Nonhematologic toxicity & & & \\
Nausea/vomiting grade $\geq 3$ & 5 & 4 & 0.718 \\
TRT-induced toxicity & & & \\
Esophagitis & & & \\
Grade 2 & 2 & - & - \\
Grade 3 & 0 & - & - \\
Pneumonitis & & & \\
Grade 2 & 2 & - & - \\
Grade 3 & 1 & - & - \\
Grade 4 & 0 & - & - \\
Grade 5 & 0 & - & - \\
\hline
\end{tabular}

ChT, chemotherapy; ChT/TRT, chemotherapy combined with thoracic radiation therapy.

that radiotherapy at 50.4 Gy in 28 fractions daily resulted in survival rates comparable with those of split-course twice-daily radiotherapy at 24 Gy in 16 fractions, a 2.5-week break and 24 Gy in 16 fractions (31). In the current study, the median PFS rate was 11 months in the 20 patients receiving TRT at $45 \mathrm{~Gy} / 30$ fractions twice per day, and 9 months in the 22 patients receiving radiotherapy at $60 \mathrm{~Gy} / 30$ fractions daily $(\mathrm{P}=0.043)$; however, no significant difference was detected in the OS rate between the two radiotherapy regimens. Thus, TRT delivered at $45 \mathrm{~Gy} / 30$ fractions twice daily appears feasible for the treatment of patients with ES-SCLC.

Consolidative TRT at $40 \mathrm{~Gy} / 15$ fractions, $45 \mathrm{~Gy} / 30$ fractions delivered twice daily and $36 \mathrm{~Gy} / 12$ fractions has been found to result in 1- and 2-year locoregional failure rates of 26 and 39\%, 1- and 2-year distant failure rates of 58 and 74\%, a median OS time of 14 months, and 1- and 2-year OS rates of 58 and $14 \%$ in patients with ES-SCLC with minimal metastatic disease, respectively. Consolidative TRT is therefore shown to control the locoregional disease in the majority of patients with minimal acute toxicity, though distant failure remains a significant problem (32). In the present study, SCLC progression and distant metastasis were observed in 23 and 39 patients in the ChT/TRT group, and 41 and 35 patients in the ChT group, respectively. A higher local control of SCLC was observed in the ChT/TRT group compared with the ChT group (48.8 vs. $28 \% ; \mathrm{P}=0.006$ ), while no significant difference in the distant control was found between the two groups $(\mathrm{P}>0.05)$.

The results of the present study showed that receiving $\geq 4$ cycles of chemotherapy improved OS and PFS, while no further benefit was achieved from chemotherapy for $>6$ cycles. These results support the hypothesis that 4-6 cycles of chemotherapy, administered either alone or concurrently with radiotherapy, may be an appropriate choice for the treatment of patients with ES-SCLC. This hypothesis has been validated by a previous study (33).

Concurrent radiochemotherapy administered at an early stage has been shown to improve local control and survival rates in patients with LS-SCLC (9); however, the optimum timing of TRT administration for patients with ES-SCLC remains unclear. In the Shandong Cancer Hospital, TRT is usually administered to patients with ES-SCLC who respond to therapy following $>2$ cycles of chemotherapy. In the current study, TRT was initiated following 2 cycles of chemotherapy in 32 subjects, 4 cycles in 13 subjects and 6 cycles in 13 subjects. Such therapies resulted in comparable OS and PFS rates with those observed in the entire ChT/TRT group, which indicated that the timing of TRT addition has no effect on treatment efficacy in ES-SCLC. However, it is suggested that TRT should be administered at a late stage of integrated therapy to ensure that more patients complete the initial 4-6 cycles of chemotherapy.

In general, ES-SCLC patients with metastasis in a single organ are given radiotherapy following chemotherapy, while those with metastasis in $>1$ organ are recommended to receive chemotherapy with radiotherapy used only as a palliative treat- 
ment (28). In the present retrospective study, 64 patients in the ChT/TRT group and 47 patients in the ChT group had metastasis in a single organ. A significant difference was detected in the number of metastatic organs between the two groups $(\mathrm{P}=0.005)$. Further prospective, multicenter and randomized trials are required to evaluate the value of TRT in the treatment of ES-SCLC patients with metastasis in one organ.

PCI, which has been found to reduce the incidence rate of symptomatic brain metastases and prolong disease-free survival and OS times, is now recommended for patients with ES-SCLC with CR to chemotherapy (34). In the present retrospective study, PCI was only administered to 5 patients with a CR to chemotherapy. Thus, the association between PCI and survival rate was not evaluated.

During SCLC therapy, treatment-induced toxicity was carefully monitored. Hematologic toxicity was the most common adverse reaction observed, with leukopenia at grades 3 or 4 in 47 patients $(57.3 \%)$ in the ChT/TRT group and 29 patients $(34.9 \%)$ in the ChT group $(\mathrm{P}=0.004)$. No significant differences were detected in the occurrence of other toxicities between the two groups, including thrombocytopenia, anemia, nausea and vomiting. Three patients had pneumonitis (grade 2 in 2 patients and grade 3 in 1 patient) in the ChT/TRT group, with a lower occurrence rate of radiation pneumonitis observed as compared with that found in a previous study (35).

In conclusion, the present study found that the addition of TRT improved the OS and PFS of patients with ES-SCLC. It is thus cautiously recommended that 4-6 cycles of standard chemotherapy followed by TRT at 45 Gy/30 fractions twice daily may be an appropriate treatment protocol for patients with ES-SCLC.

\section{References}

1. Herbst RS, Heymach JV and Lippman SM: Lung cancer. N Engl J Med 359: 1367-1380, 2008.

2. van Meerbeeck JP, Fennell DA and De Ruysscher DK: Small-cell lung cancer. Lancet 378: 1741-1755, 2011.

3. Simon GR and Wagner H; American College of Chest Physicians: Small cell lung cancer. Chest 123 (Suppl): 259S-271S, 2003.

4. Jett JR, Schild SE, Kesler KA and Kalemkerian GP: Treatment of small cell lung cancer: Diagnosis and management of lung cancer 3rd ed: American College of Chest Physicians evidence-based clinical practice guidelines. Chest 143 ( 5 Suppl): e400S-e419S, 2013.

5. Zhu H,Zhou Z, Wang Y, et al: Thoracic radiation therapy improves the overall survival of patients with extensive-stage small cell lung cancer with distant metastasis. Cancer 117: 5423-5431, 2011.

6. Yee D, Butts C, Reiman A, et al: Clinical trial of post-chemotherapy consolidation thoracic radiotherapy for extensive-stage small cell lung cancer. Radiother Oncol 102: 234-238, 2012.

7. Pignon JP, Arriagada R, Ihde DC, Johnson DH, Perry MC, Souhami RL, et al: A meta-analysis of thoracic radiotherapy for small-cell lung cancer. N Engl J Med 327: 1618-1624, 1992.

8. Warde P and Payne D: Does thoracic irradiation improve survival and local control in limited-stage small-cell carcinoma of the lung? A meta-analysis. J Clin Oncol 10: 890-895, 1992.

9. Takada M, Fukuoka M, Kawahara M, et al: Phase III study of concurrent versus sequential thoracic radiotherapy in combination with cisplatin and etoposide for limited-stage small-cell lung cancer: Results of the Japan Clinical Oncology Group Study 9104. J Clin Oncol 20: 3054-3060, 2002.

10. Simon GR and Turrisi A; American College of Chest Physicians: Management of small cell lung cancer: ACCP evidence-based clinical practice guidelines (2nd edition). Chest 132 (3 Suppl): 324-339, 2007.

11. National Comprehensive Cancer Network: Prostate cancer. NCCN Clinical practice guidelines in oncology. J Natl Compr Canc Netw 2: 224-248, 2004.
12. Faivre-Finn $\mathrm{C}$, Lorigan $\mathrm{P}$, West $\mathrm{C}$ and Thatcher N: Thoracic radiation therapy for limited-stage small-cell lung cancer: Unanswered questions. Clin Lung Cancer 7: 23-29, 2005.

13. Eisenhauer EA, Therasse P, Bogaerts J, et al: New response evaluation criteria in solid tumours: Revised RECIST guideline (version 1.1). Eur J Cancer 45: 228-247, 2009.

14. Siegel R, Naishadham D and Jemal A: Cancer statistics, 2013. CA Cancer J Clin 63: 11-30, 2013.

15. Hoffman PC, Mauer AM and Vokes EE: Lung cancer. Lancet 355: 479-485, 2000.

16. Samson DJ, Seidenfeld J, Simon GR, et al: Evidence for management of small cell lung cancer: ACCP evidence-based clinical practice guidelines (2nd edition). Chest 132 (3 Suppl): 314S-323S, 2007.

17. Cooper S and Spiro SG: Small cell lung cancer: treatment review. Respirology 11: 241-248, 2006.

18. Kallianos A, Rapti A, Zarogoulidis P, Tsakiridis K, Mpakas A, Katsikogiannis N, et al: Therapeutic procedure in small cell lung cancer. J Thorac Dis 5 (Suppl 4): S420-S424, 2013.

19. Ganti AK, West WW and Zhen W: Current concepts in the management of small cell lung cancer. Indian J Med Res 137: 1043-1051, 2013.

20. Califano R, Abidin AZ, Peck R, Faivre-Finn C and Lorigan P: Management of small cell lung cancer: recent developments for optimal care. Drugs 72: 471-490, 2012.

21. Simon M, Argiris A and Murren JR: Progress in the therapy of small cell lung cancer. Crit Rev Oncol Hematol 49: 119-133, 2004

22. National Comprehensive Cancer Network. NCCN Clinical Practice Guidelines in Oncology: Small Cell Lung Cancer. v.2, 2011. www.nccn.org: Accessed May 21, 2011.

23. Früh M, De Ruysscher D, Popat S, Crinò L, Peters S and Felip E; ESMO Guidelines Working Group: Small-cell lung cancer (SCLC): ESMO clinical practice guidelines for diagnosis, treatment and follow-up. Ann Oncol 24 (Suppl 6): vi99-vi105, 2013.

24. Noda K, Nishiwaki Y, Kawahara M, et al: Irinotecan plus cisplatin compared with etoposide plus cisplatin for extensive small-cell lung cancer. N Engl J Med 346: 85-91, 2002.

25. Hanna N, Bunn PA Jr, Langer C, et al: Randomized phase III trial comparing irinotecan/cisplatin with etoposide/cisplatin in patients with previously untreated extensive-stage disease small-cell lung cancer. J Clin Oncol 24: 2038-2043, 2006.

26. Natale RB, Lara PN, Chansky K, Crowley JJ, Jett JR, Carleton JE et al: S0124: A randomized phase III trial comparing irinotecan/cisplatin (IP) with etoposide/cisplatin (EP) in patients (pts) with previously untreated extensive stage small cell lung cancer (E-SCLC). J Clin Oncol 26 (Suppl): 7512, 2008.

27. Toy E, Macbeth F, Coles B, Melville and Eastwood A: Palliative thoracic radiotherapy for non-small-cell lung cancer: A systematic review. Am J Clin Oncol 26: 112-120, 2003.

28. Jeremic B, Shibamoto Y, Nikolic N, et al: Role of radiation therapy in the combined-modality treatment of patients with extensive disease small-cell lung cancer: A randomized study. J Clin Oncol 17: 2092-2099, 1999.

29. Turrisi AT III, Kim K, Blum R, Sause WT, Livingston RB, Komaki R, et al: Twice-daily compared with once-daily thoracic radiotherapy in limited small-cell lung cancer treated concurrently with cisplatin and etoposide. N Engl J Med 340: 265-271, 1999.

30. Watkins JM, Fortney JA, Wahlquist AE, Shirai K, Garrett-Mayer E, Aguero EG, et al: Once-daily radiotherapy to $>$ or $=59.4 \mathrm{~Gy}$ versus twice-daily radiotherapy to $>$ or $=45.0 \mathrm{~Gy}$ with concurrent chemotherapy for limited-stage small-cell lung cancer: a comparative analysis of toxicities and outcomes. Jpn J Radiol 28: 340-348, 2010.

31. Schild SE, Bonner JA, Shanahan TG, et al: Long-term results of a phase III trial comparing once-daily radiotherapy with twice-daily radiotherapy in limited-stage small-cell lung cancer. Int J Radiat Oncol Biol Phys 59: 943-951, 2004.

32. Giuliani ME, Atallah S, Sun A, et al: Clinical outcomes of extensive stage small cell lung carcinoma patients treated with consolidative thoracic radiotherapy. Clin Lung Cancer 12: 375-379, 2011.

33. Schiller JH, Adak S, Cella D, et al: Topotecan versus observation after cisplatin plus etoposide in extensive-stage small-cell lung cancer: E7593 - a phase III trial of the Eastern Cooperative Oncology Group. J Clin Oncol 19: 2114-2122, 2001.

34. Slotman B, Faivre-Finn C, Kramer G, Rankin E, Snee M, Hatton M, et al: Prophylactic cranial irradiation in extensive small-cell lung cancer. N Engl J Med 357: 664-672, 2007

35. Roeder F, Friedrich J, Timke C, Kappes J, Huber P, Krempien R, Debus J and Bischof M: Correlation of patient-related factors and dose-volume histogram parameters with the onset of radiation pneumonitis in patients with small cell lung cancer. Strahlenther Onkol 186: 149-156, 2010. 